\title{
RESONANT SCATTERING AND RECOMBINATION IN CAL 87
}

\author{
J. Greiner, ${ }^{1}$ A. Iyudin, ${ }^{1,2}$ M. Jimenez-Garate,${ }^{3}$ V. Burwitz, ${ }^{1}$ R. Schwarz,${ }^{4}$ R. DiStefano, ${ }^{5}$ and N. Schulz ${ }^{3}$
}

\section{RESUMEN}

Se observó la binaria eclipsante de rayos X supersuave CAL 87 con Chandra el 13/14 de agosto de 2001, durante casi $100 \mathrm{ksec}$, completando dos ciclos orbitales totales y tres eclipses. La forma de la curva de luz del eclipse derivada de los fotones de orden cero indica que el tamaño de la región de emisión de rayos X es de alrededor de $1.5 \mathrm{R}_{(\odot)}$. El espectro ACIS/LETG está dominado por completo por las líneas de emisión sin ningún continuo apreciable. Las líneas de emisión más brillantes presentan un corrimiento al rojo significativo y dos picos, sugiriendo que se producen en un viento de $2000 \mathrm{~km} / \mathrm{s}$. Realizamos un modelo del espectro de rayos $\mathrm{X}$ mezclando la dispersión de recombinación y resonancia. Esto nos permite deducir que la temperatura y la luminosidad de la fuente ionizante son $k T \sim 50-100 \mathrm{eV}$ y $L_{X} \sim 5 \times 10^{37} \mathrm{erg} / \mathrm{s}$, respectivamente.

\section{ABSTRACT}

The eclipsing supersoft X-ray binary CAL 87 has been observed with Chandra on August 13/14, 2001 for nearly $100 \mathrm{ksec}$, covering two full orbital cycles and three eclipses. The shape of the eclipse light curve derived from the zeroth-order photons indicates that the size of the $\mathrm{X}$-ray emission region is about $1.5 \mathrm{R}_{\odot}$. The ACIS/LETG spectrum is completely dominated by emission lines without any noticeable continuum. The brightest emission lines are significantly redshifted and double-peaked, suggestive of emanating in a $2000 \mathrm{~km} / \mathrm{s}$ wind. We model the X-ray spectrum by a mixture of recombination and resonant scattering. This allows us to deduce the temperature and luminosity of the ionizing source to be $k T \sim 50-100 \mathrm{eV}$ and $L_{X} \sim 5 \times 10^{37} \mathrm{erg} / \mathrm{s}$.

\section{Key Words: BINARIES: CLOSE - STARS: INDIVIDUAL (CAL 87) - X-RAY: STARS}

\section{INTRODUCTION}

CAL 87 was detected with the IPC onboard the Einstein X-ray observatory during the Columbia Astrophysical Laboratory survey of LMC (Long et al. 1981), and it was optically identified with an eclipsing binary in the Large Magellanic Cloud with an orbital period of $10.6 \mathrm{hrs}$ (Pakull et al. 1988, Callanan etal. 1989, Cowley etal. 1990). A shallow X-ray eclipse was discovered with ROSAT (Schmidtke et al. 1993).

Early attempts to model the optical light curve of CAL 87 by Callanan et al. (1989) already indicated an elevated accretion disk rim. This model has been extended by Schandl et al. (1997), and successfully describes the optical light curve of the CAL 87 binary system, composed of a primary (WD) with a mass of $M_{1}=0.75 \mathrm{M}_{\odot}$, placed at a distance of $2.2 \times 10^{11} \mathrm{~cm}$ from the mass-donating secondary star with a mass of $\mathrm{M}_{2}=1.5 \mathrm{M}_{0}$. The model of Schandl et al. (1997) includes (1) optical emission from the secondary star which is irradiated by the emission of the WD and (2) emission of an accretion disk with a thick rim

\footnotetext{
${ }^{1}$ MPE Garching, 85741 Garching, Germany.

${ }^{2}$ Moscow, Russia.

${ }^{3}$ MIT, Cambridge, MA 02139, USA.

${ }^{4}$ Universität Göttingen, 37083 Germany.

${ }^{5} \mathrm{CfA}$, Cambridge, MA 02138, USA.
}

and an optically thick, cold, clumpy spray produced by the high mass-flow rate of the accretion stream impinging on the disk (hot spot). This spray, moving around the disk, nicely reproduced the asymmetry in the optical light curve and the depth of the secondary dip.

Based on the pre-Chandra, low-resolution X-ray spectra and the detection of emission up to $1 \mathrm{keV}$, CAL 87 has long been considered as one of the two hottest known SSS, thus spurring observations with all X-ray satellites since ROSAT (Parmar et al. 1997, Asai et al. 1998, Dotani et al. 2000, Ebisawa et al. 2001).

We have exploited the unique spectral capability of the Chandra low-energy transmission grating (LETG) to study in detail the X-ray emission of the canonical supersoft source CAL 87.

\section{CHANDRA OBSERVATION IN 2001}

We observed CAL 87 with Chandra for $97 \mathrm{ksec}$ on August 13/14, 2001, using the low-energy transmission grating (LETG/ACIS). This observation covered three consecutive eclipses of the binary system. The phase-folded light curve of the zeroth order photons (Fig. 1) shows a wide eclipse with triangular shape, about \pm 0.15 phase units wide $(30 \%$ of the orbit), no flat bottom and an eclipse depth of about 


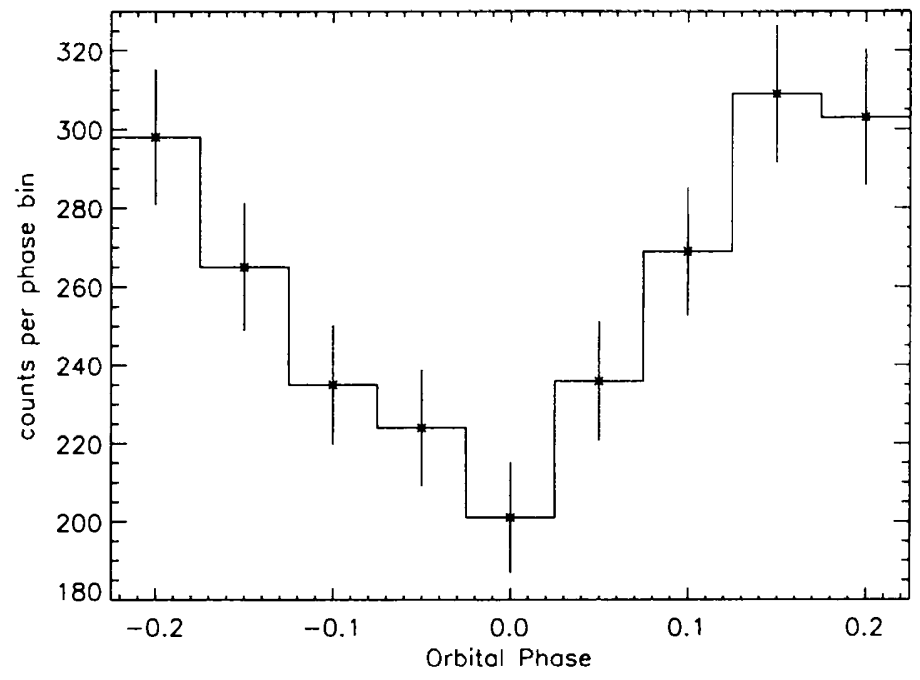

Fig. 1. Phase-folded light curve of the zeroth order events around the eclipse minimum, showing the broad width and triangular shape of the eclipse.

$50 \%$. This suggests that the observed X-ray emission does not come from a point source (e.g. the $\mathrm{H}$-burning white dwarf). Instead, the occulter and the eclipsed X-ray emission region should have about equal size. Given the orbital period and assuming that the donor star is filling its Roche lobe $\left(1.5 \mathrm{R}_{\odot}\right)$, the size of the emission region should be $\sim 1.5 \mathrm{R}_{\odot}$ (at a binary separation of $3.5 \mathrm{R}_{\odot}$ ).

The spectrum of CAL 87 is completely dominated by emission lines (Fig. 2), predominantly from oxygen, nitrogen and iron. Hardly any continuum emission is detectable. Galactic foreground and LMC-intrinsic absorption cuts off the spectrum above $\sim 25 \AA$. The extinction-corrected X-ray luminosity is $\sim 10^{35} \mathrm{erg} / \mathrm{s}$. No emission is detected shortward of $14 \AA$ (corresponding to $>0.9 \mathrm{keV}$ ), suggesting that the effective temperature of the primary (expected to be thermal) X-ray emission near the accreting object is smaller than $\sim 80 \mathrm{eV}$.

One example for the plasma diagnostic constraints which can be derived is the OVII line at $21.7 \AA$ : the strong resonance line and the absence of the forbidden and intercombination lines indicates either collisional excitation or resonance scattering. The prominence of the $17.05 / 17.10 \AA$ lines compared to the other Fe XVII lines argues for a significant contribution from recombination emission. Also, the presence of higher Ly series emission as well as the lack of the Fe XVII $15.01 \AA$ and $16.78 \AA$ emission lines (as compared to the strong Fe XVII 17.05/17.10 $\AA$ lines) argue against collisional excitation. Thus, as also supported by other line ratios, the X-ray spectrum of CAL 87 is mixture of recombination emission and resonant scattering.

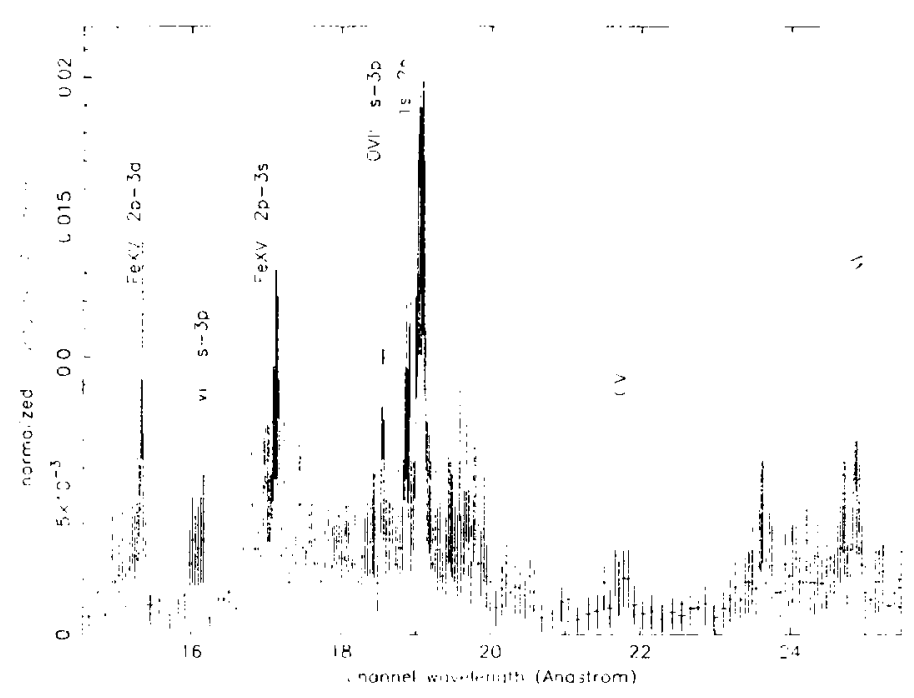

Fig. 2. Chandra LETG/ACIS count spectrum of C'AL 87 summed over the bright phase (excluding the eclipse). Identifications of strong emission lines are given.

We have begun modelling the X-ray emission of CAL 87 by computing the emergent spectrum produced by à central source of $k T=65 \mathrm{eV}, L_{\mathrm{X}}$ $=5 \times 10^{37} \mathrm{erg} / \mathrm{s}$, ionizing and being scattered off a corona with an electron density of $10^{12} \mathrm{~cm}^{-3}$ and log $\xi=3.8$ (see Fig. 3). While the observed global features can be reproduced, a higher fraction of scattering is needed. A first examination of the parameter space indicates that this can be achieved with either a softer ionizing continuum and/or a lower column density.

An interesting property of the brightest emission line, O VIII Lya $(18.97 \AA)$, is that it is redshifted and has a double-peaked profile. The same behaviour is also noticable in the next-brightest lines (N VII, O VII), though with lower significance. The shift in wavelength is constant, and also does not vary with orbital phase. In addition, the red peak disappears during eclipse. We interpret this as a wind/outflow with an observed velocity of 1200 and $2200 \mathrm{~km} / \mathrm{s}$, respectively, after taking into account the systemic LMC velocity. The disappearance of the red peak can be understood if the wind/outflow is not spherically symmetric, but a bi-directional cone. If we assume intrinsic velocity symmetry of both sides of the cone, and de-project for the inclination of $\sim 78^{\circ}$, we obtain an opening angle of the conical wind/outflow of $120^{\circ}$. With such a geometry, the northern/upper part of the wind/outflow is predominantly blue-shifted, and the southern/lower part redshifted. During eclipse, only the southern part of the wind/outflow will be occulted by the donor, an observed. 

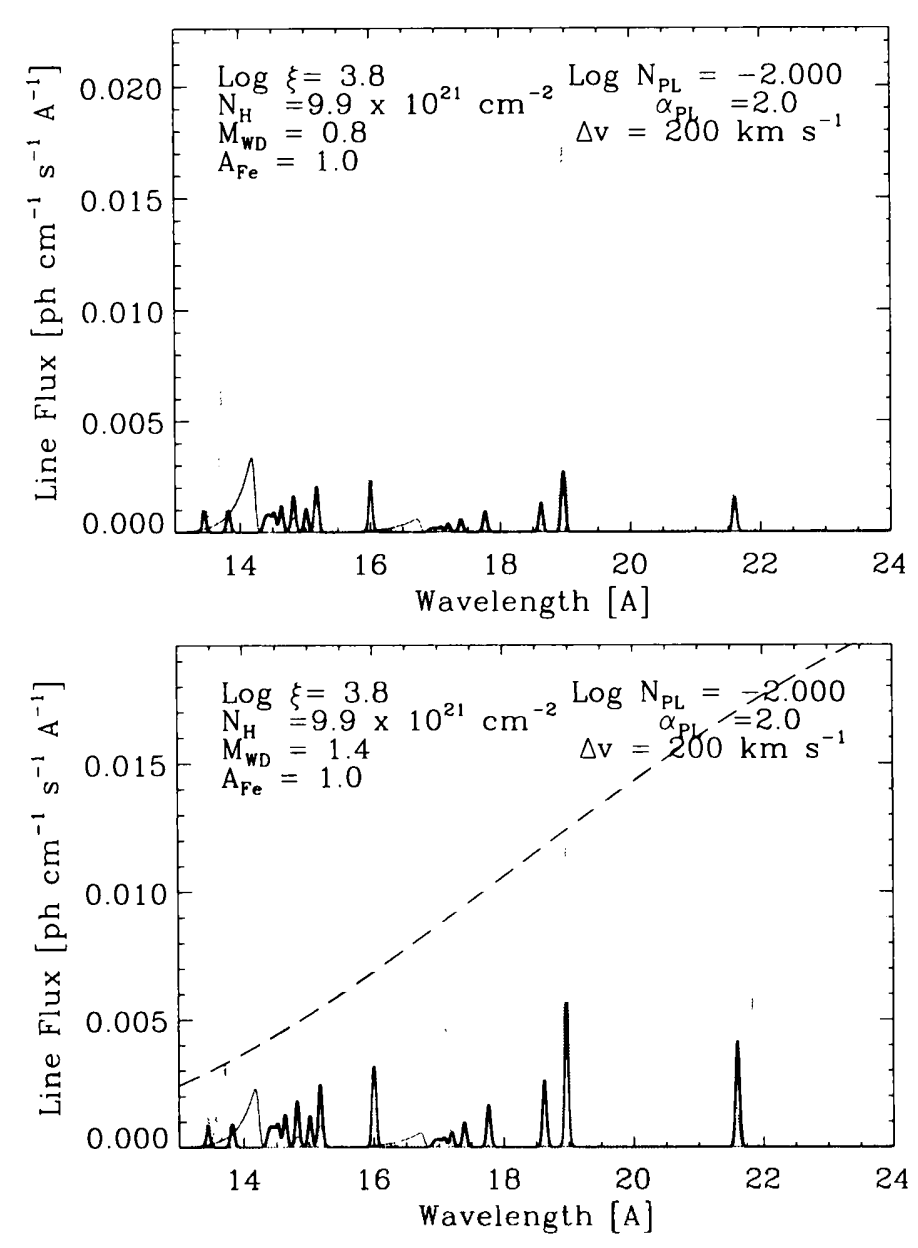

Fig. 3. Preliminary model of the X-ray spectrum of CAL 87 , composed of recombination (blue) and resonant scattering (black) with a white dwarf mass of $0.8 \mathrm{M}_{\odot}$ (top) and $1.4 \mathrm{M}_{\odot}$ (bottom), corresponding to different effective temperatures of the ionizing source. The dashed line in the lower panel is the primary white dwarf spectrum.

\section{CONCLUSIONS}

The shape of the eclipse light curve, the lack of any continuum emission and the dominance of emission lines due to resonant scattering and recombination suggests that CAI 87's X-ray emission comes from an extended wind (or outflow), similar to the accretion-disk-corona low-mass X-ray binary $4 \mathrm{U}$ 1822-37, as already suggested by Schmidtke (1993). However. the plasma in $4 \mathrm{U} 1822-37$ (i) has a much higher ionization as evidenced by the $\mathrm{H}$ - and He-like ions of $\mathrm{Ne}, \mathrm{Mg}, \mathrm{Si}, \mathrm{S}$ and Fe which are completely absent in CAL 87 and (ii) is much more dominated by recombination, indicative of larger column densities than those in CAL 87.
The wind is probably not spherical, but rather has a conical shape with an opening angle of $120^{\circ}$, extending on both sides of the accretion disk. During eclipse, the donor occults the wind on the southern disk side.

The primary X-ray emission region in CAL 87 certainly has a temperature substantially lower than $\sim 1 \mathrm{keV}$ due to the lack of Fe L, Si XIII or Ne IX/Ne $\mathrm{X}$ lines. This rules out a neutron star or black hole (except for retrograde rotation) accretor, since they would exhibit a maximum temperature of the inner part of the accretion disk of $0.9-1.2 \mathrm{keV}$. Also, the outflow velocity of $\sim 2000 \mathrm{~km} / \mathrm{s}$ is consistent with a white dwarf primary.

A corona with an ionization parameter and electron density ranging from $>3000 / 10^{12} \mathrm{~cm}^{-3}$ near the accretor to $\sim 30 / 10^{11} \mathrm{~cm}^{-3}$ near the outer boundary would scatter about $1 \%$ of the irradiated X-ray emission. This suggests that the intrinsic X-ray luminosity of CAL 87 is of order a few times $10^{37} \mathrm{erg} / \mathrm{s}$.

Though the earlier SSS classification was based on a wrong interpretation of the X-ray spectrum, the new $\mathrm{kT}$ and L estimates from our LETG spectrum re-confirm the supersoft source nature of CAL 87.

JG and RD gratefully acknowledge the Chandra Award No. GO1-2022X.

\section{REFERENCES}

Asai, K., Dotani, T., Nagase, F., Ebisawa, K., Mukai, K. et al. 1998, ApJ 503, L143

Callanan P.J., Machin G., Naylor T., Charles P.A. 1989, MNRAS 241, 37p

Cowley A.P., Schmidtke P.C., Crampton D., Hutchings J.B. 1990, ApJ 350, 288

Dotani T., Asai K., Ebisawa K., Greiner J., 2000, COSPAR, Adv. Space Res. 25, No. 3/4, 375

Ebisawa K., Mukai K., Kotani T., et al. 2001, ApJ 550, 1007

Long K.S., Helfand D.J., Grabelsky D.A. 1981, ApJ 248, 925

Pakull M.W., Beuermann K., van der Klis M., van Paradijs J., 1988, A\&A 203, L27

Parmar A.N., Kahabka P., Hartmann H.W. et al., 1997, A\&A 323, L33

Schandl S., Meyer-Hofmeister E., Meyer F., 1997, A\&A 318,73

Schmidtke P.C., McGrath T.K., Cowley A.P., Frattare L.M. 1993, PASP 105, 863

Vadim Burwitz, Jochen Greiner and Anatoli Iyudin: Max-Planck-Institut für extraterrestrische Physik,

Giessenbachstraße, 85748 Garching, Germany (burwitz,jcg,ani@mpe.mpg.de).

Rosanne Di Stefano: Center for Astrophysics, Cambridge, MA 02138. U.S.A. (rd@cfa.harvard.edu).

Mario Jimenez-Garate and Norbert Schulz: MIT, Cambridge, U.S.A. (mario,nss@space.mit.edu).

Robert Schwarz: Universität Göttingen, 37083 Göttingen, Germany, (rsc@uni-sw.gwdg.de). 